\title{
A prospective observational study of comparison of tympanoplasty using endomeatal approach using tragal cartilage and perichondrium with post auricular approach using temporalis fascia graft: an institutional study
}

\author{
V. Krishna Chaitanya*, N. Janardhan, Teja, Vinay Singh Bhat, Rajaram \\ Department of ENT, Narayana Medical College and Hospital, Nellore, Andhra Pradesh, India \\ Received: 10 November 2017 \\ Revised: 26 November 2017 \\ Accepted: 27 November 2017

\section{*Correspondence:} \\ Dr. V. Krishna Chaitanya, \\ E-mail: drvkc17@gmail.com \\ Copyright: (c) the author(s), publisher and licensee Medip Academy. This is an open-access article distributed under \\ the terms of the Creative Commons Attribution Non-Commercial License, which permits unrestricted non-commercial \\ use, distribution, and reproduction in any medium, provided the original work is properly cited.
}

\begin{abstract}
Background: Evaluation of success of tympanoplasty depends on various host and surgical factors has been subject of interest for many years and still continues to be challenge.

Methods: Present study was undertaken to observe results of type I tympanoplasty using tragal cartilage and perichondrium with temporalis fascia grafts using endomeatal and postauricular approach.

Results: Study group comprises of 30 patients in Group A and B operated by tympanoplasty type I by endomeatal and postauricular techniques. Success was $96.3 \%$ in Group A and $94.7 \%$ in Group B for closure of membrane perforation. During postoperative period average time taken for wound healing was 23 days in Group A and 35 days in Group B. Preoperative and postoperative assessment of hearing performed with pure tone audiometry revealed an average hearing loss of $36.50 \mathrm{~dB}$ in Group A before surgery and postoperative assessment at end of 1 year revealed hearing improvement of $14.23 \mathrm{~dB}$. In Group B preoperative assessment revealed hearing loss of $38.23 \mathrm{~dB}$ lowered to $15.12 \mathrm{~dB}$ after surgery. When hearing improvement in both groups were compared preoperatively and postoperatively, in Group A there was improvement of $22.27 \mathrm{~dB}$ while in Group B was $23.11 \mathrm{~dB}$. The hearing improvements in both the groups appear to be similar when the results were compared. In present study overall success rate was $89.7 \%$ which is comparable to outcome of surgery performed with post auricular approach with temporalis fascia graft which is $91.3 \%$. When postoperative complications were studied it was observed that Group A had fewer numbers of complications as over Group B which was documented in Table 2.

Conclusions: Success of tympanoplasty depends on several host and surgical factors. Tragal perichondrium appears as proven alternate for closure of perforation of tympanic membrane. Cosmetically endomeatal approach appears promising over post auricular approach.
\end{abstract}

Keywords: Tympanoplasty, Fascia, Cartilage, Audiometry, Pure-tone

\section{INTRODUCTION}

Although tympanoplasty is being preferred as the procedure of choice for the eradication of disease in middle ear with closure of tympanic membrane perforation and is also helpful in reconstruction of the hearing mechanism with or without ossiculoplasty, there exists a lot of interest in various techniques of tympanoplasty and their clinical outcomes. ${ }^{1}$ Though a lot of work has been published in this area and research is progressing to a lot more advanced aspects, understanding the proper technique of approach of the 
surgery of tympanoplasty, the technique and the clinical outcomes of the surgery is always essential for a clinician in ENT.

Evaluation of the surgical success of tympanoplasty an assessment of various host and surgical factors has been a subject of interest for many years and still continues to be a challenge. ${ }^{2}$ To have a quantitative measurement of subjects hearing and to give scientific credibility to results of the clinical tests an audiometric evaluation can be done. $^{3}$ Comparison of the preoperative and postoperative pure tone averages in speech frequencies and the air-bone gap gives a complete picture of the improvement in hearing after surgery.

The present study was undertaken to evaluate and compare the results of type I tympanoplasty using temporalis fascia and tragal cartilage and perichondrium grafts with respect to dry ear and hearing status using postauricular and endomeatal approach. The present study emphasizes on the role of the approach and procedure in planning appropriate management for the patient with chronic suppurative otitis media.

\section{Objectives}

To assess the outcome of surgical technique and determine the hearing status before and after tympanoplasty using endomeatal approach using tragal cartilage and perichondrium with post auricular approach using temporalis fascia graft in the patients with chronic perforation of the tympanic membrane.

\section{METHODS}

In the present study, a total of 60 patients with chronic suppurative otitis media with a dry perforation of tympanic membrane with a hearing loss up to $45 \mathrm{~dB}$ during February 2013 to January 2014 were included. All these patients selected for the study were selected considering the inclusion and exclusion criteria to the study following a standard clinical protocol.

\section{Inclusion and exclusion criteria}

Patients with chronic suppurative otitis media tubotympanic disease having dry perforated tympanic membrane in the age between 16 to 60 years with good cochlear reserve having hearing loss up to $45 \mathrm{~dB}$ were included in the study.

Patients presenting with hearing loss greater than $45 \mathrm{~dB}$, immunocompramised patients, patients presenting with sensorineural hearing loss, patients presenting with marginal perforation, atticoantral disease, tympanosclerosis and otosclerosis were excluded from the study.

These selected patients were evaluated with an appropriate clinical questionnaire and a thorough clinical examination was performed and the exact nature of the disease was confirmed. These patients were further examined by microscopy and pure tone audiometry was performed. All selected patients for surgery were examined and screened with laboratory investigations. A written informed consent was obtained from all the patients for performing the procedure.

Patients were divided into two groups comprising of 30 patients in each group. In Group A tympanoplasty was performed with endomeatal technique using tragal cartilage and perichondrium and in Group B tympanoplasty was performed using post auricular approach with temporalis fascia graft and the results were observed. All the surgeries were performed observing a standard protocol. Canaloplasty was performed to improve the visualisation of the surgical field in patients of both the groups as and where required. All the surgeries were performed by a single surgeon who has adequate surgical experience in performing the ear surgeries of tympanoplasty.

All the patients were followed up for a period of 1 year and the results were assessed. All these patients were followed up at the end of one week, four and six weeks postoperatively. During these visits a microscopic examination of the ears was performed and the status of their postoperative graft was recorded. The final follow up was carried out at the end of 1 year after the surgery and microscopic examinations were performed, along with pure tone audiometry to determine the final state of graft and assess the hearing status of the patient and the results of the surgical procedure were observed.

\section{RESULTS}

In the present study out of 60 patients 23 patients $(55.0 \%)$ were females and $27(45.0 \%)$ were males. The number of male patients in Group A was 14 (46.66\%) and that of the female patients was $16(53.33 \%)$. Meanwhile, among the patients in Group B, $13(43.33 \%)$ were male and 17 $(56.66 \%)$ were female. Amongst the 60 patients involved in the study, $34(56.67 \%)$ had a central perforation of tympanic membranes and $26(43.33 \%)$ had subtotal perforation.

From the 30 people in Group A, 16 (53.33\%) had central perforation and $14(46.67 \%)$ had medium to subtotal perforation. In contrast, among the patients in Group B $18(60.0 \%)$ had central perforation and $12(40.0 \%)$ had medium to subtotal perforation. The overall success rate of tympanoplasty in our study was $96.3 \%$ in Group A and $94.7 \%$ in Group B in terms of closing the membrane perforation.

It was observed that during the postoperative period the average time taken for the healing of wound was 23 days in patients of Group A and the average time taken for wound healing was 35 days in patients of Group B. From the time taken for the wound to heal it was observed that 
in Group A the average time taken for wound healing was less over the Group B.

In patients of Group A preoperative and postoperative assessment of hearing was performed using pure tone audiometry and air bone gap was measured which revealed an average hearing loss of $36.50 \mathrm{~dB}$ in patients in Group A before the surgery. When hearing was assessed post operatively at the end of 1 year the hearing improvement was observed having an average hearing of $14.23 \mathrm{~dB}$. An improvement in hearing was observed after the surgery in Group A.
In patients of Group B preoperative and postoperative assessment of hearing was performed using pure tone audiometry and air bone gap was measured which revealed a hearing loss of $38.23 \mathrm{~dB}$ in patients before the surgery, which was lowered to $15.12 \mathrm{~dB}$ after the surgery. When the hearing improvement in both the groups were compared preoperatively and postoperatively by pure tone audiometry the average hearing improvement in all Group A patients before and after the surgery was 22.27 $\mathrm{dB}$ while those in Group B was $23.11 \mathrm{~dB}$. The hearing improvements in both the groups appear to be similar when the results were compared. The assessment of AB gap was documented in the Table 1.

Table I: Assessment of AB gap.

\begin{tabular}{|c|c|c|c|c|c|c|c|c|}
\hline & \multicolumn{4}{|l|}{ Group A } & \multicolumn{4}{|l|}{ Group B } \\
\hline & \multicolumn{2}{|c|}{ Preoperative } & \multicolumn{2}{|c|}{ Postoperative } & \multicolumn{2}{|c|}{ Preoperative } & \multicolumn{2}{|c|}{ Postoperative } \\
\hline & Number & $\%$ & Number & $\%$ & Number & $\%$ & Number & $\%$ \\
\hline 0-10 & 0 & 0 & 5 & 16.66 & 0 & 0 & 7 & 23.33 \\
\hline 11-20 & 7 & 23.33 & 23 & 76.67 & 5 & 16.67 & 20 & 66.67 \\
\hline 21-30 & 23 & 76.67 & 2 & 6.67 & 25 & 83.33 & 3 & 10.0 \\
\hline$>\mathbf{3 0}$ & 0 & 0 & 0 & 0 & 0 & 0 & 0 & 0 \\
\hline Total & 30 & 100.0 & 30 & 100.0 & 30 & 100.0 & 30 & 100.0 \\
\hline
\end{tabular}

Table 2: Postoperative complications.

\begin{tabular}{|lllll|}
\hline & Group A & & Group B & Number \\
\hline Number & \% & 1 & 3.33 \\
\hline Suture gaping & 0 & 0 & 1 & 3.33 \\
\hline Delayed wound healing & 0 & 0 & 1 & 3.33 \\
\hline Wound site infection & 0 & 0 & 1 & 3.33 \\
\hline Graft failure & 2 & 6.67 & 3 & 10.0 \\
\hline
\end{tabular}

In the present study the average overall success rate was $89.7 \%$ which is comparable to the outcome of surgery performed with post auricular approach with temporalis fascia graft which is $91.3 \%$. When the postoperative complications were studied it was observed that Group A had fewer numbers of complications as over Group B which was documented in the Table 2.

\section{DISCUSSION}

The success of tympanoplasty is assessed from graft uptake, hearing improvement, complications intraoperative and postoperative. ${ }^{4}$ The most commonly accepted steps for optimum success of tympanoplasty include resection of the margins of perforations, raising large tympanomeatal flaps, and the graft placement between the bony wall of the canal and the large bloody flap. ${ }^{5}$

Quite a number of factors affect the outcome of surgical results of tympanoplasty and a substantial amount of research work has been carried out regarding the impact of various factors such as age, sex, perforation size, the status of the opposite ear, discharge status of the ear at the time of surgery, surgical approaches and techniques, and the materials used for the graft.

After the evolution of the surgical technique of tympanoplasty temporalis fascia is proved to be a better graft material for the procedure owing to its easy approach to harvest and similarities with the natural tympanic membrane and similarly post auricular approach is considered to be a better approach as it has advantages of being performed easily with an access to approach the critical areas of the ear and beyond. ${ }^{6}$ This approach proved to be a promising approach till date. In the present study the overall success rate of tympanoplasty with regard to graft uptake and improvement in hearing was $91.3 \%$ in patients of Group A where tympanoplasty was performed with endomeatal approach with tragal perichondrium graft.

In the present day era of decreased morbidity with surgery and owing to the cosmetic importance of a small scar and early recovery newer approaches for tympanoplasty are developed. The endomeatal approach is emerging as a dependant technique owing to the 
reduced morbidity and improved cosmesis. ${ }^{7}$ This technique can be easily performed for small posterior perforations but appears challenging for significant anterior perforations, narrow or stenotic ear canals, or individuals with a significant anterior canal bulge. When this approach is combined with canaloplasty for the improvement of visualisation of the tympanic membrane the results of this technique can be promising. ${ }^{8}$

With the advent of endomeatal approach for tympanoplasty and cosmetic awareness, tragal cartilage with perichondrium is recently being used as a graft in tympanoplasty. In the present study it was observed that graft uptake rate was slightly better for temporalis fascia (96.3\%) than for tragal perichondrium (94.7\%). The results of tympanoplasty with temporalis fascia were equal to that with tragal perichondrium, when graft uptake is concerned.

Given these results, we might conclude that tragal perichondrium appears to be a better alternative to temporalis fascia as graft for tympanoplasty taking hearing improvement and graft uptake together. temporalis fascia and tragal perichondrium are excellent graft material for closure of perforation of tympanic membrane and hearing improvement. Both the post auricular and endomeatal approaches of tympanoplasty provide satisfying results. Owing to the cosmesis and early wound healing endomeatal approach can be considered as a proven alternative over the conventional technique of tympanoplasty.

\section{CONCLUSION}

The success of tympanoplasty depends on several host and surgical factors. Tragal perichondrium appears as a proven alternate for closure of perforation of tympanic membrane and hearing improvement. Adequate selection of patient and surgical experience plays a role in success of the surgery where the cosmetic appealing endomeatal approach appears promising equally with post auricular approach of tympanoplasty.
Funding: No funding sources Conflict of interest: None declared

Ethical approval: The study was approved by the Institutional Ethics Committee

\section{REFERENCES}

1. Demirpehlivan IA, Onal K, Arslanoglu S, Songu M, Ciger E, Can N. Comparison of different tympanic membrane reconstruction techniques in type I tympanoplasty. Eur Arch Oto-Rhino-Laryngol. 2011;268(3):471-4.

2. Levin B, Rajkhowa R, Redmond SL, Atlas MD. Grafts in myringoplasty: utilizing a silk fibroin scaffold as a novel device. Expert Rev Med Devices. 2009;6(6):653-64.

3. Atherley G, Johnston N. Audiometry-the ultimate test of success? Annals Occupational Hygiene. 1983;27(4):427-47.

4. Dornhoffer J. Cartilage tympanoplasty: Indications, techniques, and outcomes in A 1,000-patient series. The Laryngoscope. 2003;113(11):1844-56.

5. Tarabichi M. Endoscopic transcanal middle ear surgery. Indian J Otolaryngol Head Neck Surg. 2010;62(1):6-24.

6. Sheehy JL, Anderson RG. Myringoplasty: a review of 472 cases. Annals Otol Rhinol Laryngol. 1980;89(4):331-4.

7. Coskun BU, Cinar U, Seven H, Ugur S, Dadas B. The effects of the incision types in myringoplasty operations on cosmesis. Eur Arch Oto-RhinoLaryngol Head Neck. 2006;263(9):820-2.

8. Jang JH, Song JJ, Yoo JC, Lee JH, Oh SH, Chang SO. An alternative procedure for cochlear implantation: transcanal approach. Acta otolaryngologica. 2012;132(8):845-9.

Cite this article as: Chaitanya VK, Janardhan N, Teja, Bhat VS, Rajaram. A prospective observational study of comparison of tympanoplasty using endomeatal approach using tragal cartilage and perichondrium with post auricular approach using temporalis fascia graft: an institutional study. Int J Otorhinolaryngol Head Neck Surg 2018;4:41-4. 